\title{
The very local Hubble flow ${ }^{\star, \star \star}$
}

\author{
I. D. Karachentsev ${ }^{1}$, M. E. Sharina ${ }^{1,10}$, D. I. Makarov ${ }^{1,10}$, A. E. Dolphin ${ }^{2}$, E. K. Grebel ${ }^{3}$, D. Geisler ${ }^{4}$, \\ P. Guhathakurta ${ }^{5}$, P. W. Hodge ${ }^{6}$, V. E. Karachentseva ${ }^{7}$, A. Sarajedini ${ }^{8}$, and P. Seitzer ${ }^{9}$ \\ 1 Special Astrophysical Observatory, Russian Academy of Sciences, N. Arkhyz, KChR, 369167, Russia \\ 2 Kitt Peak National Observatory, National Optical Astronomy Observatories, PO Box 26732, Tucson, AZ 85726, \\ USA \\ 3 Max-Planck-Institut für Astronomie, Königstuhl 17, 69117 Heidelberg, Germany \\ 4 Departamento de Física, Grupo de Astronomía, Universidad de Concepción, Casilla 160-C, Concepción, Chile \\ ${ }^{5}$ UCO/Lick Observatory, University of California at Santa Cruz, Santa Cruz, CA 95064, USA \\ ${ }^{6}$ Department of Astronomy, University of Washington, Box 351580, Seattle, WA 98195, USA \\ 7 Astronomical Observatory of Kiev University, 04053, Observatorna 3, Kiev, Ukraine \\ 8 Department of Astronomy, University of Florida, Gainesville, FL 32611, USA \\ 9 Department of Astronomy, University of Michigan, 830 Dennison Building, Ann Arbor, MI 48109, USA \\ 10 Isaac Newton Institute, Chile, SAO Branch
}

Received 24 December 2001 / Accepted 25 April 2002

\begin{abstract}
We present Hubble Space Telescope/WFPC2 images of eighteen galaxies situated in the vicinity of the Local Group (LG) as part of an ongoing snapshot survey of nearby galaxies. Their distances derived from the magnitude of the tip of the red giant branch are $1.92 \pm 0.10 \mathrm{Mpc}$ (ESO 294-010), $3.06 \pm 0.37$ (NGC 404), $3.15 \pm 0.32$ (UGCA 105), $1.36 \pm 0.07$ (Sex B), $1.33 \pm 0.08$ (NGC 3109), $2.64 \pm 0.21$ (UGC 6817), $2.86 \pm 0.14$ (KDG 90), $2.27 \pm 0.19$ (IC 3104), $2.54 \pm 0.17$ (UGC 7577), $2.56 \pm 0.15$ (UGC 8508), $3.01 \pm 0.29$ (UGC 8651), $2.61 \pm 0.16$ (KKH 86), 2.79 \pm 0.26 (UGC 9240), 1.11 \pm 0.07 (SagDIG), $0.94 \pm 0.04$ (DDO 210), $2.07 \pm 0.18$ (IC 5152), $2.23 \pm 0.15$ (UGCA 438), and $2.45 \pm 0.13$ (KKH 98). Based on the velocity-distance data for 36 nearest galaxies around the LG, we find the radius of the zero-velocity surface of the LG to be $R_{0}=(0.94 \pm 0.10)$ Mpc, which yields a total mass $M_{\mathrm{LG}}=(1.3 \pm 0.3) \times 10^{12} M_{\odot}$. The galaxy distribution around the LG reveals a Local Minivoid which does not contain any galaxy brighter than $M_{V}=-11 \mathrm{mag}$ within a volume of $\sim 100 \mathrm{Mpc}^{3}$. The local Hubble flow seems to be very cold, having a one-dimensional mean random motion of $\sim 30 \mathrm{~km} \mathrm{~s}^{-1}$. The best-fit value of the local Hubble parameter is $73 \pm 15 \mathrm{~km} \mathrm{~s}^{-1} \mathrm{Mpc}^{-1}$. The luminosity function for the nearby field galaxies is far less steep than one for members of the nearest groups.
\end{abstract}

Key words. galaxies: dwarf - galaxies: distances and redshifts - galaxies: kinematics and dynamics galaxies: Local Group

\section{Introduction}

Until quite recently the behaviour of the Hubble flow in the immediate vicinity of the Local Group (LG) remained practically unknown because of the lack of reliable data on distances to many nearest galaxies. Lynden-Bell (1981) and Sandage (1986) demonstrated that the velocitydistance relation for galaxies around the LG should be non-linear due to gravitational deceleration produced by the LG mass. Being determined from observational data,

\footnotetext{
Send offprint requests to: M. E. Sharina, e-mail: sme@sao.ru

* Figure 2 is only available in the electronic form at http://www. edpsciences.org

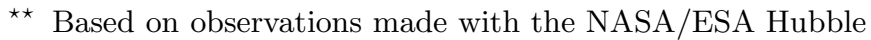
Space Telescope. The Space Telescope Science Institute is operated by the Association of Universities for Research in Astronomy, Inc. under NASA contract NAS 5-26555.
}

the effect of deceleration permits calculation of the total mass of the LG independently from mass estimates based on virial/orbital motions. In the distance range of $(1 \div 3) \mathrm{Mpc}$, where the expected non-linearity of the Hubble relation is significant, Sandage (1986) used velocities and distances of seven galaxies: Leo A $(1.58 \mathrm{Mpc})$, IC 5152 (1.58), NGC 3109 (1.58), NGC 300 (1.66), Sex A (1.74), Sex B (1.74), and Pegasus (2.51 Mpc) and estimated the LG mass to be $4 \times 10^{11} M_{\odot}$. As became clear later, the distances of two of these seven galaxies (Leo A and Pegasus) were incorrect by more than a factor of 2. Based on largely the same observational data, Giraud $(1986,1990)$ obtained a three times higher mass for the LG. Karachentsev \& Makarov (2001) used accurate distance measurements for 20 galaxies in the vicinity of the LG and determined the radius of the zerovelocity sphere, $R_{0}$, which separates the $\mathrm{LG}$ from the 
total cosmological expansion. From their data $R_{0}$ is of $0.96 \pm 0.05 \mathrm{Mpc}$. According to Lynden-Bell (1981), this radius corresponds to the total LG mass, $M_{\mathrm{LG}}=\left(\pi^{2} / 8 G\right)$. $H_{0}^{2} \cdot R_{0}^{3}$, where $H_{0}$ is the local Hubble parameter and $G$ is the gravitational constant. For $H_{0}=70 \mathrm{~km} \mathrm{~s}^{-1} \mathrm{Mpc}^{-1}$ the radius of $0.96 \mathrm{Mpc}$ yields a total mass of $1.2 \times 10^{12} M_{\odot}$. Karachentsev \& Makarov (2001) also noted that the dispersion of peculiar radial velocities of galaxies around the LG is surprisingly small, only $\sim 25 \mathrm{~km} \mathrm{~s}^{-1}$.

Over the last few years, rapid progress has been made in accurate distance measurements for nearby galaxies beyond the Local Group. At present, the number of galaxies with known distances within $(1 \div 3)$ Mpc is approaching $\sim 40$. As a result of searching for new nearby objects on the POSS-II/ESO-SERC plates (Karachentseva et al. 1998, 1999; Karachentsev et al. 2001a), a number of low surface brightness dwarf galaxies have recently been found. The local galaxy complex candidates are included in the program of our snapshot survey with the Hubble Space Telescope (HST; programs GO-8192, GO-8601, Seitzer et al. 1999). We have reported the discovery of two very nearby dwarf galaxies, KK 3 and KKR 25, situated within 2 Mpc (Karachentsev et al. 2000, 2001b). Below we present new distance measurements for 18 more nearby galaxies observed in the snapshot survey.

\section{WFPC2 photometry and data reduction}

The galaxies were imaged with the Wide Field and Planetary Camera (WFPC2) during 1999 October 15 to 2001 July 28 as part of our HST snapshot survey of nearby galaxy candidates (Seitzer et al. 1999). The objects were usually centered on the WF3 chip, but for some bright galaxies the WFPC2 position was shifted towards the galaxy periphery to decrease the stellar crowding effect. 600-second exposures were taken in the F606W and F814W filters for each object. Digital Sky Survey images (DSS-II, red) of the eighteen galaxies are shown in Fig. 1 with the HST WFPC2 footprints superimposed. The field size of the DSS images is $10^{\prime} \times 10^{\prime}$.

The photometric reduction was carried out using the HSTphot stellar photometry package described by Dolphin (2000a). After removing cosmic rays with the HSTphot cleansep routine, simultaneous photometry was performed on the $F 606 \mathrm{~W}$ and $F 814 \mathrm{~W}$ frames using multiphot, with aperture corrections for an aperture of radius 0.5 . Charge-transfer efficiency (CTE) corrections and calibrations were then applied, based on the Dolphin (2000b) formulae, producing $V I$ photometry for all stars detected in both images. Because of the relatively small field of the Planetary Camera (PC) chip, very few bright stars are available for the computation of the aperture correction. Thus the PC photometry was omitted in further analysis. Additionally, stars with the signal-to-noise ratio $S / N<5,|\chi|>2.0$, or $\mid$ sharpness $\mid>0.4$ in each exposure were eliminated from the final photometry list. We estimate the uncertainty of the photometric zeropoint to be within $0{ }^{\mathrm{m}} 05$ (Dolphin 2000b).
The WFPC2 images of the galaxies are presented in upper panels of Fig. 2, where both filters are combined. The compass in each field indicates the North and East directions.

\section{Color-magnitude diagrams and distances}

The middle panels of Fig. 2 show $I$ versus $(V-I)$ colormagnitude diagrams (CMDs) for the eighteen observed galaxies. As demonstrated by Lee et al. (1993), the tip of the red giant branch (TRGB) is a reliable distance indicator that is relatively independent of age and metallicity. In the $I$ band the TRGB for low-mass stars is found to be stable within $\sim 0.1 \mathrm{mag}$ (Salaris \& Cassisi 1996; Udalski et al. 2001) for metallicities, $[\mathrm{Fe} / \mathrm{H}]$, encompassing the entire range from -2.1 up to -0.7 represented by Galactic globular clusters. According to Da Costa \& Armandroff (1990), for metal-poor systems the TRGB is located at $M_{I}=-4.05 \mathrm{mag}$. Also, Lee et al. (1993) derived the TRGB to be in the range of $-4.0 \pm 0.1$ mag. Ferrarese et al. (2000) calibrated a zero point of the TRGB from galaxies with Cepheid distances, and yielded $M_{I}=-4.06 \pm 0.07$ (random $) \pm 0.13$ (systematic) mag. A new TRGB calibration $M_{I}=-4.04 \pm 0.12 \mathrm{mag}$ was determined by Bellazzini et al. (2001) based on photometry and a distance estimate from a detached eclipsing binary in the Galactic globular cluster $\omega$ Centauri. For this paper we use the calibration of $M_{I}=-4.05 \mathrm{mag}$.

To determine the TRGB location, we obtained a Gaussian-smoothed $I$-band luminosity function for red stars with colors $V-I$ within \pm 0 m 5 of the mean $\langle V-I\rangle$ for expected red giant branch (RGB) stars. Following Sakai et al. (1996), we used a Sobel edge-detection filter. The position of the TRGB was identified with the peak in the filter response function. The resulting luminosity functions and the Sobel-filtered luminosity functions are shown in the bottom panels of Fig. 2. The results are summarized in Table 1. The data listed in its columns are as follow: (1) galaxy name; (2) equatorial coordinates corresponding to the galaxy center; $(3,4)$ apparent integrated magnitude and angular dimension from the NASA Extragalactic Database (NED); (5) heliocentric radial velocity; (6) morphological type in de Vaucouleurs notation; (7) position of the TRGB and its uncertainty derived with the Sobel filter; (8) Galactic extinction in the $I$ - band (Schlegel et al. 1998); (9) true distance modulus with its uncertainty, which takes into account the uncertainty in the TRGB detection, as well as uncertainties of the HST photometry zero point $\left(\sim 0 .{ }^{\mathrm{m}} 05\right)$, the aperture corrections $\left(\sim 0{ }^{\mathrm{m}} 05\right)$, and crowding effects $\left(\sim 0{ }^{\mathrm{m}} 06\right)$ added in quadrature; the uncertainties in extinction and reddening are taken to be $10 \%$ of their values from Schlegel et al. (1998); (more details on the total budget of internal and external systematic errors for the TRGB method see in Mendez et al. 2002); and (10) linear distance in Mpc and its uncertainty. Some individual properties of the galaxies are discussed below. 


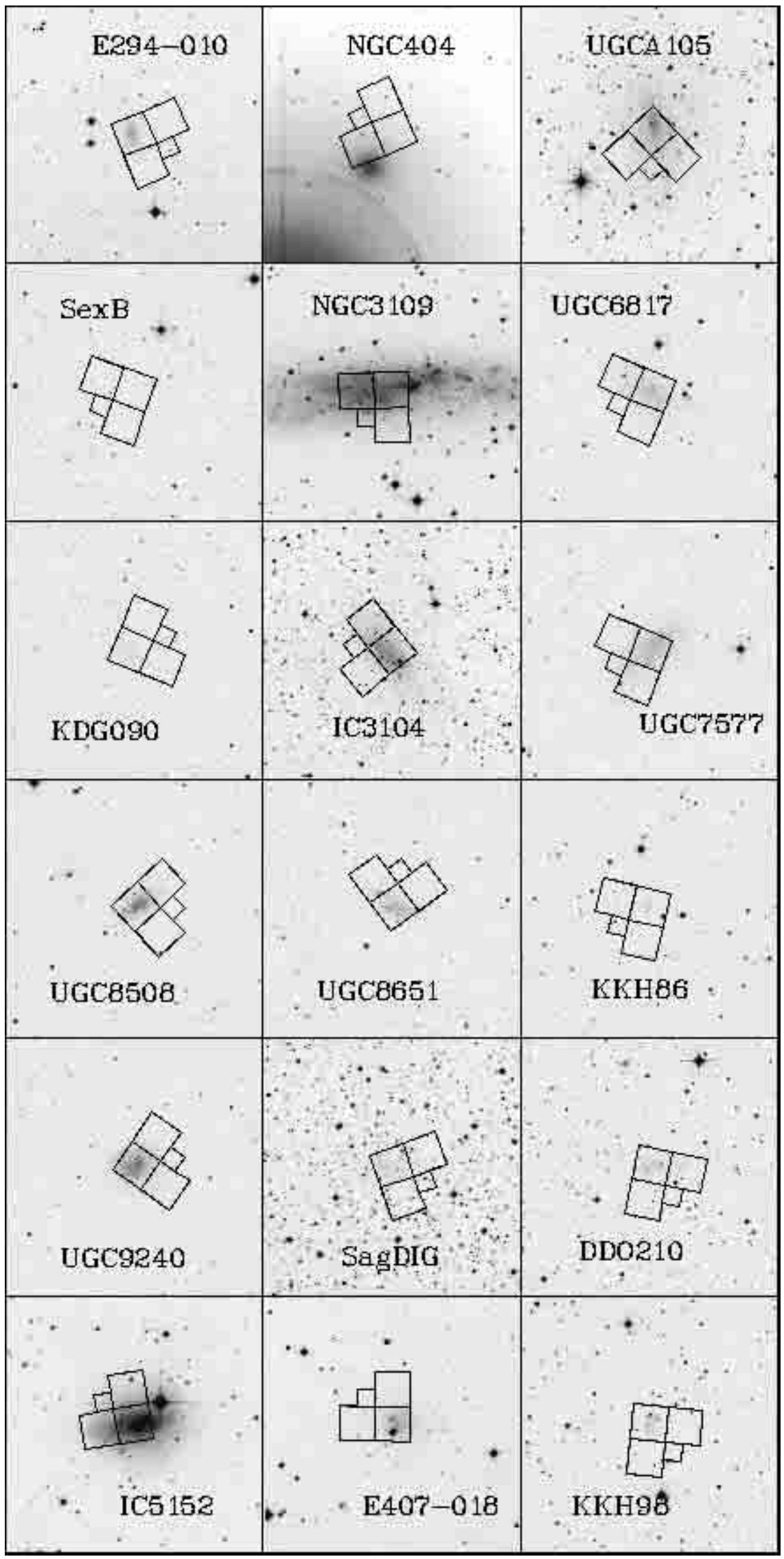

Fig. 1. Digital Sky Survey images of 18 galaxies in the vicinity of the Local Group. The field size is $10^{\prime}$, North is up and East is left. The HST WFPC2 footprints are superimposed. 
Table 1. New distances to very nearby galaxies around the Local Group.

\begin{tabular}{|c|c|c|c|c|c|c|c|c|c|}
\hline Name & $\begin{array}{l}\alpha(1950.0) \delta^{\alpha} \\
\text { hhmmss } \\
\text { ○० } \prime \prime \prime \prime \prime \prime\end{array}$ & $\begin{array}{r}B_{\mathrm{T}} \\
\mathrm{mag}\end{array}$ & $\begin{array}{r}a \times b \\
\operatorname{arcmin}\end{array}$ & $\begin{array}{r}V_{\mathrm{h}} \pm \sigma \\
\mathrm{km} \mathrm{s}^{-1}\end{array}$ & $\bar{T}$ & $\begin{array}{r}I \text { (TRGB) } \\
\text { mag }\end{array}$ & $\begin{array}{r}A_{I} \\
\mathrm{mag}\end{array}$ & $\begin{array}{r}(m-M)_{0} \\
m a g\end{array}$ & $\begin{array}{r}D \\
\mathrm{Mpc}\end{array}$ \\
\hline ESO294-010 & $002406.2-420755$ & 15.60 & $1.1 \times 0.7$ & $117 \pm 5$ & $-1:$ & 22.38 & 0.01 & 26.42 & 1.92 \\
\hline PGC 01641 & & & & & & 0.05 & & .10 & .10 \\
\hline NGC 404 & 010639.2352705 & 11.21 & $3.5 \times 3.5$ & $-48 \pm 9$ & -1 & $\begin{array}{r}23.49 \\
.24\end{array}$ & 0.11 & $\begin{array}{r}27.43 \\
.25\end{array}$ & $\begin{array}{r}3.06 \\
.37\end{array}$ \\
\hline UGCA 105 & 050935.6623431 & 13.9 & $5.5 \times 3.5$ & $111 \pm 5$ & 10 & $\begin{array}{r}24.05 \\
.20\end{array}$ & 0.61 & $\begin{array}{r}27.49 \\
.22\end{array}$ & $\begin{array}{r}3.15 \\
.32\end{array}$ \\
\hline $\begin{array}{l}\text { Sex B } \\
\text { DDO } 070\end{array}$ & 095723.1053421 & 11.85 & $5.1 \times 3.5$ & $301 \pm 1$ & 10 & $\begin{array}{r}21.72 \\
.09\end{array}$ & 0.06 & $\begin{array}{r}25.66 \\
.13\end{array}$ & $\begin{array}{r}1.36 \\
.07\end{array}$ \\
\hline NGC 3109 & $100049.0-255504$ & 10.39 & $19.7 \times 3.7$ & $403 \pm 1$ & 10 & $\begin{array}{r}21.70 \\
.10\end{array}$ & 0.13 & $\begin{array}{r}25.62 \\
.13\end{array}$ & $\begin{array}{r}1.33 \\
.08\end{array}$ \\
\hline UGC 6817 & 114816.8390930 & 13.4 & $4.1 \times 1.5$ & $242 \pm 1$ & 10 & 23.11 & 0.05 & 27.11 & 2.64 \\
\hline DDO 099 & & & & & & .15 & & .17 & .21 \\
\hline KDG 090 & 121227.1362948 & 15.40 & $1.5 \times 1.3$ & $284 \pm 6$ & $-1:$ & 23.27 & 0.04 & 27.28 & 2.86 \\
\hline DDO 113 & & & & & & .06 & & .11 & .14 \\
\hline IC 3104 & $121545.0-792654$ & 13.63 & $3.8 \times 1.8$ & $430 \pm 5$ & 9 & $\begin{array}{r}23.49 \\
.16\end{array}$ & 0.76 & $\begin{array}{r}26.78 \\
.18\end{array}$ & $\begin{array}{r}2.27 \\
.19\end{array}$ \\
\hline $\begin{array}{l}\text { UGC } 7577 \\
\text { DDO } 125\end{array}$ & 122515.4434613 & 12.84 & $4.3 \times 2.4$ & $196 \pm 4$ & 10 & $\begin{array}{r}23.01 \\
.10\end{array}$ & 0.04 & $\begin{array}{r}27.02 \\
.13\end{array}$ & $\begin{array}{r}2.54 \\
.17\end{array}$ \\
\hline $\begin{array}{l}\text { UGC } 8508 \\
\text { I Zw060 }\end{array}$ & 132847.1551002 & 14.40 & $1.7 \times 1.0$ & $62 \pm 5$ & 10 & $\begin{array}{r}23.02 \\
.09\end{array}$ & 0.03 & $\begin{array}{r}27.04 \\
.13\end{array}$ & $\begin{array}{r}2.56 \\
.15\end{array}$ \\
\hline $\begin{array}{l}\text { UGC } 8651 \\
\text { DDO } 181\end{array}$ & 133744.2405931 & 14.7 & $2.3 \times 1.3$ & $201 \pm 1$ & 10 & $\begin{array}{r}23.35 \\
.19\end{array}$ & 0.01 & $\begin{array}{r}27.39 \\
.21\end{array}$ & $\begin{array}{r}3.01 \\
.29\end{array}$ \\
\hline KKH 86 & 135202.2042917 & 16.8 & $0.7 \times 0.5$ & $283 \pm 3$ & 10 & $\begin{array}{r}23.08 \\
.10\end{array}$ & 0.05 & $\begin{array}{r}27.08 \\
.13\end{array}$ & $\begin{array}{r}2.61 \\
.16\end{array}$ \\
\hline $\begin{array}{l}\text { UGC } 9240 \\
\text { DDO } 190\end{array}$ & 142248.4444504 & 13.25 & $1.8 \times 1.6$ & $150 \pm 4$ & 10 & $\begin{array}{r}23.20 \\
.18\end{array}$ & 0.02 & $\begin{array}{r}27.23 \\
.20\end{array}$ & $\begin{array}{r}2.79 \\
.26\end{array}$ \\
\hline SagDIG & $192705.4-174659$ & 14.12 & $2.9 \times 2.1$ & $-77 \pm 4$ & 10 & $\begin{array}{r}21.26 \\
.03\end{array}$ & 0.22 & $\begin{array}{r}25.09 \\
.10\end{array}$ & $\begin{array}{r}1.04 \\
.05\end{array}$ \\
\hline $\begin{array}{l}\text { DDO } 210 \\
\text { Aquarius }\end{array}$ & $204406.5-130155$ & 14.0 & $2.2 \times 1.1$ & $-137 \pm 5$ & 10 & $\begin{array}{r}20.91 \\
.05\end{array}$ & 0.10 & $\begin{array}{r}24.86 \\
.10\end{array}$ & $\begin{array}{r}0.94 \\
.04\end{array}$ \\
\hline IC 5152 & $215926.6-513214$ & 11.06 & $5.2 \times 3.2$ & $124 \pm 3$ & 10 & $\begin{array}{r}22.58 \\
.16\end{array}$ & 0.05 & $\begin{array}{r}26.58 \\
.18\end{array}$ & $\begin{array}{r}2.07 \\
.18\end{array}$ \\
\hline $\begin{array}{l}\text { ESO407-018 } \\
\text { UGCA } 438\end{array}$ & $232347.3-323950$ & 13.86 & $1.5 \times 1.2$ & $62 \pm 5$ & 10 & $\begin{array}{r}22.72 \\
.12\end{array}$ & 0.03 & $\begin{array}{r}26.74 \\
.15\end{array}$ & $\begin{array}{r}2.23 \\
.15\end{array}$ \\
\hline KKH 98 & 234303.9382624 & 16.7 & $1.1 \times 0.6$ & $-136 \pm 3$ & 10 & $\begin{array}{r}23.14 \\
.06\end{array}$ & 0.24 & $\begin{array}{r}26.95 \\
.11\end{array}$ & $\begin{array}{r}2.45 \\
.13 \\
\end{array}$ \\
\hline
\end{tabular}

ESO 294-010 = PGC 01641. The galaxy has a smooth regular shape and the integrated color $(B-R)_{\mathrm{T}}=$ 1.17 (Jerjen et al. 1998), typical of dwarf spheroidal (dSph) galaxies. These authors also measured the distance to the galaxy, $1.71 \pm 0.07 \mathrm{Mpc}$, via fluctuations of surface brightness and found weak emission in [O III] and $\mathrm{H} \alpha$ with a radial velocity of $+117 \pm 5 \mathrm{~km} \mathrm{~s}^{-1}$. Our CMD (Fig. 2) shows the presence of a small number of faint blue stars situated in the central and southern parts of the galaxy, which confirms the classification of the galaxy as a dwarf system of mixed type, like Phoenix, LGS 3, and KK 3. Judging by its velocity and distance, ESO 294-010 is a probable companion of the spiral galaxy NGC 55 .

NGC 404. This compact lenticular galaxy with dusty patches in its central part is partially affected by the halo of a bright star. Tonry et al. (2001) determined its distance as $3.26 \pm 0.15 \mathrm{Mpc}$ based on fluctuations of its surface brightness. NGC 404 is the nearest representative of the rare class of isolated S0 galaxies. There are no other known galaxies within 1.1 Mpc around it (Karachentsev \& Makarov 1999). The origin of such an object is unclear. This galaxy may represent the final stage of consecutive merging of members of a former group of galaxies.

UGCA 105. This irregular galaxy probably belongs to the Maffei/IC 342 group situated in a zone of strong Galactic extinction. Tikhonov et al. (1992) and Karachentsev et al. (1997) resolved it into stars and determined its distance from the luminosity of the brightest stars to be $3.3 \mathrm{Mpc}$ and $3.2 \mathrm{Mpc}$, respectively. The distance, $3.15 \pm 0.32 \mathrm{Mpc}$, that we have derived agrees well with these estimates.

Sextans B. According to Sakai et al. (1997) the distance to this dIrr galaxy found from the luminosity of its RGB stars is $1.29 \pm 0.07 \mathrm{Mpc}$. Our estimate of its distance, $1.36 \pm 0.07 \mathrm{Mpc}$, agrees well with the previous one.

NGC 3109. Capaccioli et al. (1992) studied Cepheids in this galaxy and derived from them a distance of $1.26 \pm 0.10 \mathrm{Mpc}$. Our distance estimate from the TRGB, 
$1.33 \pm 0.08 \mathrm{Mpc}$, agrees within the uncertainties with the Cepheid distance. According to Tully et al. (2002), NGC 3109 together with three other dwarf galaxies, Sex A, Sex B, and Antlia, form a loose group of "squelched" galaxies in a common dark halo.

UGC $6817=$ DDO 99. This dIrr galaxy has been resolved into stars for the first time by Georgiev et al. (1997), who determined its distance as $3.9 \pm 0.8 \mathrm{Mpc}$ via the brightest stars. Our distance estimate from the TRGB yields a lower distance, $2.64 \pm 0.21 \mathrm{Mpc}$, which corresponds to the galaxy location on the front side of the Canes Venatici cloud.

K $90=$ DDO $113=$ UGCA 276. This dwarf galaxy of low surface brightness and of regular smooth shape was resolved into stars by Makarova et al. (1997). Jerjen et al. (2002) determined its integrated color $(B-R)=$ $1.07 \mathrm{mag}$ and the central surface brightness $\Sigma_{\mathrm{B}}(0)=$ $24.92 \pm 0.08 \mathrm{mag} \operatorname{arcsec}^{-2}$. These authors estimated the galaxy distance as $3.1 \pm 0.3 \mathrm{Mpc}$ from surface brightness fluctuations, which agrees well with our estimate, $2.86 \pm 0.14 \mathrm{Mpc}$, from the TRGB. As one can see from its CMD (Fig. 2), K 90 does not contain a significant number of blue stars, which favours this dwarf's classification as a dSph system. However, the galaxy shows strong HI emission, which makes us regard it as a mixed dSph/dIrr system. Being situated $10^{\prime}$ away from the bright spiral galaxy NGC 4214, K 90 is a probable companion of the galaxy because their velocities differ from each other by only $9 \mathrm{~km} \mathrm{~s}^{-1}$. Like UGC $6817, \mathrm{~K} 90$ is situated on the front side of the CVn cloud.

IC $3104=$ ESO 20-004 = PGC 039573. As far as we know this irregular galaxy with a radial velocity of only $V_{\mathrm{LG}}=171 \mathrm{~km} \mathrm{~s}^{-1}$ has now been resolved into stars for the first time. The distance, $2.27 \pm 0.19 \mathrm{Mpc}$, derived by us is consistent with its low radial velocity. IC 3104 is a clear example of how poorly the immediate vicinity of the LG has so far been studied.

UGC 7577 = DDO 125. This dIrr galaxy was resolved into stars by Tikhonov \& Karachentsev (1998), who estimated its distance to be $4.8 \pm 1.0 \mathrm{Mpc}$ via the brightest stars. The TRGB position (Fig. 2) yields a much lower distance, $2.54 \pm 0.17 \mathrm{Mpc}$, and allows us to consider UGC 7577 to be situated on the front side of the CVn cloud.

UGC $8508=$ I Zw 60. Karachentsev et al. (1994) resolved this dIrr galaxy into stars and determined a distance of $3.7 \pm 0.8 \mathrm{Mpc}$ from the brightest stars. The TRGB position for UGC 8508 corresponds to a distance of $2.56 \pm 0.15 \mathrm{Mpc}$, placing the galaxy in front of the M 101 group.

UGC $8651=$ DDO 181. The galaxy has a curved bow-like shape similar to DDO 165. Its distance, $3.4 \pm$ $0.7 \mathrm{Mpc}$, was estimated by Makarova et al. (1998) via the brightest stars. The distance from its TRGB position is $3.01 \pm 0.29 \mathrm{Mpc}$. Like some above mentioned galaxies, UGC 8651 apparently belongs to the near side of the CVn cloud.
KKH 86. This dwarf irregular galaxy of low surface brightness was found by Karachentsev et al. (2001a). Surface photometry of KKH 86 has been carried out by Makarova et al. (2002). The low TRGB distance of the galaxy, $2.61 \pm 0.16 \mathrm{Mpc}$, agrees with its low velocity, $V_{\mathrm{LG}}=205 \mathrm{~km} \mathrm{~s}^{-1}$.

UGC 9240 = DDO 190. The galaxy was studied in detail by Aparicio \& Tikhonov (2000), who derived a TRGB distance of $2.9 \pm 0.2 \mathrm{Mpc}$. Our WFPC2 photometry yields almost the same distance, $2.79 \pm 0.26 \mathrm{Mpc}$. The galaxy is a probable member of the CVn cloud.

SagDIG. This dIrr galaxy is situated at a low Galactic latitude. Stellar photometry of SagDIG was made by Karachentsev et al. (1999) and Lee \& Kim (2000), who found its TRGB distance to be $1.06 \pm 0.10 \mathrm{Mpc}$ and $1.18 \pm 0.10 \mathrm{Mpc}$, respectively. Our determination of the distance, $1.04 \pm 0.05 \mathrm{Mpc}$, agrees well with both these estimates.

DDO 210 = Aquarius. Detailed stellar photometry of the galaxy was carried out by Lee et al. (1999), who derived the TRGB distance to be $0.95 \pm 0.05 \mathrm{Mpc}$. The WFPC2 photometry yields for DDO 210 the same distance, $0.94 \pm 0.04 \mathrm{Mpc}$.

IC 5152. This bright irregular galaxy contains a lot of blue stars and dusty patches. Zijlstra \& Minniti (1998) detected its TRGB and determined the distance as $1.70 \pm 0.17 \mathrm{Mpc}$. Our distance estimate is $2.07 \pm 0.18 \mathrm{Mpc}$. The lower value from Zijlstra \& Minniti may be caused by crowding effects, higher for a ground-based telescope, which tends to brighten the apparent TRGB position (e.g., Stephens et al. 2001).

UGCA $438=$ ESO 407-018. According to Lee \& Byun (1999) the TRGB distance of this irregular galaxy is $2.08 \pm 0.12 \mathrm{Mpc}$. Our WFPC2 photometry gives a slightly higher value, $2.23 \pm 0.15 \mathrm{Mpc}$. The galaxy may be a member of the known loose group in Sculptor.

KKH 98. This galaxy was found by Karachentsev et al. (2001a) as a bluish low surface brightness object partially resolved into stars. Its distance derived from the TRGB is $2.45 \pm 0.13 \mathrm{Mpc}$. KKH 98 is a well-isolated galaxy. There are no other known objects within $1 \mathrm{Mpc}$ around it.

\section{The very local velocity field}

In addition to the 18 above-mentioned galaxies, there are 20 more known galaxies situated (or may be situated) within $D \sim 3 \mathrm{Mpc}$ around the LG centroid. A summary of these objects is given in Table 2. Two galaxies from this list, KK 3, Cetus have no measured velocities yet. Another two southern galaxies, ESO 383-087 and IC 4662, have no distance estimates, but their radial velocities with respect to the LG centroid are less than $150 \mathrm{~km} \mathrm{~s}^{-1}$. For six more galaxies: SDIG, NGC 55, NGC 247, NGC 1569, UGCA 92 and UGC 8638 their distances have been determined with low accuracy. The data listed in the table columns are as follows: (1) galaxy name and cross-identification (lower line); (2) equatorial coordinates (B1950.0); (3) integrated 
apparent magnitude (upper line) and Galactic extinction from Schlegel et al. (1998) (lower line); (4) angular dimension in arcmin (upper line) and morphological type in de Vaucouleurs notation (lower line); (5) heliocentric radial velocity and its standard error in $\mathrm{km} \mathrm{s}^{-1} ;$ (6) distance to the galaxy and its standard error in Mpc; (7) distance from the LG centroid, calculated as

$D_{\mathrm{LG}}^{2}=D^{2}+\Delta^{2}-2 \cdot D \cdot \Delta \cdot \cos \theta$,

where $\Delta=0.44 \mathrm{Mpc}$ is the distance of the Milky Way from the LG centroid, and $\theta$ is the angle between the direction to the galaxy and that to $\mathrm{M} 31 ;(8)$ radial velocity of the galaxy with respect to the LG centroid with the apex parameters $V_{\mathrm{a}}=316 \mathrm{~km} \mathrm{~s}^{-1}, l_{\mathrm{a}}=93^{\circ}, b_{\mathrm{a}}=-4^{\circ}$ derived by Karachentsev \& Makarov (1996); (9) the abovementioned angle $\theta$; and (10) integrated absolute magnitude of the galaxy. The data of Cols. (2)-(5) are taken from NED, and the sources of data on distances are given in footnotes to the table.

The all-sky distribution of the 38 galaxies listed in Table 2 is presented in equatorial coordinates in Fig. 3. The distribution looks rather inhomogeneous, showing concentration of the objects towards two opposite directions: the CVn cloud and the loose Sculptor group, which confirms the location of the Local Group in a filament, extending from Canes Venatici to Sculptor (Tully 1988). Another feature of the distribution is complete absence of galaxies in a wide area around the direction towards Orion $\left(\alpha=05^{\mathrm{h}} 40^{\mathrm{m}}, \delta=-3^{\circ}\right)$. This empty region, occupying about a quarter of the entire sky, is indicated by the right-hand solid curve in Fig. 3. We nickname it as a Local Minivoid (LMV). Unlike the Local Void (Tully 1988), which has the center position about $\alpha=18^{\mathrm{h}} 38^{\mathrm{m}}, \delta=+18^{\circ}$, an angular diameter $\sim 60^{\circ}$ and a depth of $1500 \mathrm{~km} \mathrm{~s}^{-1}$ (Karachentseva et al. 1999), the LMV extends in depth to $D \sim 5 \mathrm{Mpc}$. In the about $100 \mathrm{Mpc}^{3}$, there is not a single known galaxy brighter than $M_{V}=-11^{\mathrm{m}}$. An absence of galaxies in the direction of Orion may be partially due to extinction by the Milky Way. However, the presence of such a "perfect" empty volume can not be explained by Galactic extinction alone, because some more distant dwarf galaxies ("Orion", A0554+07, UGC 3303, kk49) with radial velocities of $V_{\mathrm{LG}} \simeq 400 \mathrm{~km} \mathrm{~s}^{-1}$ are seen behind the LMV (Karachentsev \& Musella 1996). Velocities and distances of 34 galaxies from Table 2 with respect to the LG centroid are shown in Fig. 4 with indication of their observational uncertainties. As was demonstrated by Sandage $(1986,1987)$, the deceleration of the cosmic expansion in the vicinity of the LG leads to a curvature of the Hubble relation, which intersects the line of $V_{\mathrm{LG}}=0$ at a nonzero distance $R_{0}$ from the LG centroid. The value of $R_{0}$ characterizes the radius of the zero-velocity surface that separates the LG from the total cosmic expansion. In the case of spherical symmetry with the cosmological parameter $\Lambda=0$ the LG mass depends on $R_{0}$ as $M_{\mathrm{LG}}=$ $\left(\pi^{2} / 8 G\right) \cdot H_{0}^{2} \cdot R_{0}^{3}$, which yields for $H_{0}=70 \mathrm{~km} \mathrm{~s}^{-1} \mathrm{Mpc}^{-1}$ a mass $M_{\mathrm{LG}} / M_{\odot}=1.39 \times 10^{12} \cdot\left(R_{0} / \mathrm{Mpc}\right)^{3}$. In Fig. 4 the thick solid line shows the velocity-distance regression with two parameters, $H_{0}=73 \mathrm{~km} \mathrm{~s}^{-1} \mathrm{Mpc}^{-1}$ and $M_{\mathrm{LG}}=1.26 \times 10^{12} M_{\odot}$, derived as the least-square solution. The two thin solid lines correspond to the $95 \%$ confidence interval of the regression.

As one can see from these data, the majority of nearby galaxies follow the Hubble relation very well. However, a group of 7 galaxies with $D_{\mathrm{LG}} \sim 2.8 \mathrm{Mpc}$ and $V_{\mathrm{LG}} \sim$ $270 \mathrm{~km} \mathrm{~s}^{-1}$ departs appreciably from the relation as a whole. All these objects are situated on the front side of the Canes Venatici cloud and, apparently, move from us toward the cloud center at an additional velocity of about $85 \mathrm{~km} \mathrm{~s}^{-1}$. Another galaxy, NGC 6789, has the radial velocity much lower than the expected velocity. This object is situated in front of the Local Void at a high Supergalactic latitude $\left(+41.6^{\circ}\right)$. The low velocity of NGC 6789 may be caused by the gravitational action of the Local Void, which imparts a peculiar velocity of $\sim 80 \mathrm{~km} \mathrm{~s}^{-1}$ towards us on NGC 6789 . Fitting the Hubble relation with its two dependent parameters, $H_{0}$ and $M_{\mathrm{LG}}$, we omitted the seven probable members of the CVn cloud (indicated as asterisks) and also two galaxies, SDIG and NGC 247 (shown as triangles) due to their poorly determined distances. For the remaining 27 galaxies the leastsquares method yields a radius of the zero-velocity surface of $0.94 \pm 0.10 \mathrm{Mpc}$. The mean-root-square dispersion of radial velocities for field galaxies relative to the Hubble regression with the indicated parameters is $\sigma_{v}=34 \mathrm{~km} \mathrm{~s}^{-1}$. A significant part of the scatter of galaxies in Fig. 4 is caused by the uncertainties of the measured distances, $\Delta D / D \sim 10 \%$, while measurement errors of the radial velocities are relatively small, $\sim 2 \%$. Taking the errors of the observables into account, the mean-root-square random velocity of nearby galaxies themselves is reduced to $\sigma_{v}=29 \mathrm{~km} \mathrm{~s}^{-1}$.

To get a complete picture of the local velocity field, we also show in Fig. 4 the members of the Local Group, which are indicated by open circles. Observational data on their distances and velocities are listed in Table 3, the columns of which contain: (1) galaxy name; (2) morphological type, (3) measured distance and its standard error; (4) heliocentric velocity and its error; $(5,6)$ distance and radial velocity with respect to the $\mathrm{LG}$ centroid. The last two quantities are determined in the same manner as described above. The basic data are taken from the book of van den Bergh (2000) and also from Evans et al. (2000). Some of the other recent sources of distances are mentioned in the table footnotes.

Considering the structure and kinematics of two nearby groups around M 81 (Karachentsev et al. 2002a) and Centaurus A (Karachentsev et al. 2002b), we noted a general similarity of these groups with the LG. The systems of companions around M 81 and Cen A are characterized by a mean projected linear radius of $\sim 200 \mathrm{kpc}$ and a radial velocity dispersion of $\sim 80 \mathrm{~km} \mathrm{~s}^{-1}$ each. For the companions of the Milky Way and M 31, these quantities 
Table 2. Properties of very nearby galaxies at the Local Group boundaries and around the Local Group.

\begin{tabular}{|c|c|c|c|c|c|c|c|c|c|c|c|}
\hline Name & $\alpha(1950.0) \delta$ & $\begin{array}{l}B_{\mathrm{T}} \\
A_{B}\end{array}$ & $\begin{array}{c}a \times b \\
T\end{array}$ & $\overline{V_{\mathrm{h}}}$ & $\sigma$ & $\bar{D}$ & $\sigma$ & $\overline{D_{\mathrm{LG}}}$ & $\overline{V_{\mathrm{LG}}}$ & $\overline{\theta \theta}$ & $\overline{M_{B}}$ \\
\hline $\begin{array}{l}\text { SDIG } \\
\text { E349-031 }\end{array}$ & $000541.0-345124$ & $\begin{array}{r}15.48 \\
0.05\end{array}$ & $\begin{array}{c}1.1 \times 0.9 \\
10\end{array}$ & 207 & 7 & 1.6 & .8 & 1.56 & 216 & 76.2 & -10.59 \\
\hline NGC 55 & $001238.0-392954$ & $\begin{array}{l}8.84 \\
0.06\end{array}$ & $\begin{array}{c}32.4 \times 5.6 \\
9\end{array}$ & 129 & 3 & 1.66 & .4 & 1.65 & 111 & 80.7 & -17.32 \\
\hline $\begin{array}{l}\text { KK } 3 \\
\text { E410-005 }\end{array}$ & $001300.0-322736$ & $\begin{array}{r}14.85 \\
0.06\end{array}$ & $\begin{array}{c}1.3 \times 1.0 \\
-3\end{array}$ & - & & 1.92 & .19 & 1.85 & - & 73.7 & -11.63 \\
\hline $\begin{array}{l}\text { Cetus } \\
\text { KKSG } 01\end{array}$ & $002338.7-111916$ & $\begin{array}{l}14.4 \\
0.12\end{array}$ & $\begin{array}{c}5.0 \times 4.3 \\
-2\end{array}$ & - & & 0.78 & .05 & 0.62 & - & 52.4 & -10.18 \\
\hline $\begin{array}{l}\text { E294-010 } \\
\text { PGC } 01641\end{array}$ & $002406.2-420755$ & $\begin{array}{r}15.60 \\
0.02\end{array}$ & $\begin{array}{c}1.1 \times 0.7 \\
-1:\end{array}$ & 117 & 5 & 1.92 & .10 & 1.92 & 81 & 83.2 & -10.84 \\
\hline NGC 247 & $004439.6-210200$ & $\begin{array}{l}9.86 \\
0.08\end{array}$ & $\begin{array}{c}21.4 \times 6.9 \\
7\end{array}$ & 160 & 2 & 2.48 & .6 & 2.31 & 215 & 62.0 & -17.19 \\
\hline NGC 300 & $005231.8-375715$ & $\begin{array}{l}8.95 \\
0.06\end{array}$ & $\begin{array}{c}21.9 \times 15.5 \\
7\end{array}$ & 144 & 1 & 2.1 & .2 & 2.06 & 114 & 79.0 & -17.72 \\
\hline NGC 404 & 010639.2352705 & $\begin{array}{r}11.21 \\
0.25\end{array}$ & $\begin{array}{c}3.5 \times 3.5 \\
-1\end{array}$ & -48 & 9 & 3.06 & .37 & 2.62 & 195 & 7.6 & -16.47 \\
\hline NGC 1569 & 042604.6644423 & $\begin{array}{r}11.86 \\
3.02\end{array}$ & $\begin{array}{c}3.6 \times 1.8 \\
9\end{array}$ & -104 & 4 & 2.5 & .5 & 2.18 & 88 & 39.5 & -18.15 \\
\hline UGCA 92 & 042727.1633025 & $\begin{array}{r}13.8: \\
3.42\end{array}$ & $\begin{array}{c}2.0 \times 1.0 \\
10\end{array}$ & -99 & 5 & 1.8 & .5 & 1.49 & 89 & 39.5 & -15.90 \\
\hline UGCA 105 & 050935.6623431 & $\begin{array}{l}13.9 \\
1.35\end{array}$ & $\begin{array}{c}5.5 \times 3.5 \\
10\end{array}$ & 111 & 5 & 3.15 & .32 & 2.85 & 279 & 44.3 & -14.94 \\
\hline $\begin{array}{l}\text { Leo A } \\
\text { DDO } 069\end{array}$ & 095632.4305910 & $\begin{array}{r}12.92 \\
0.09\end{array}$ & $\begin{array}{c}5.1 \times 3.1 \\
10\end{array}$ & 20 & 4 & 0.69 & .06 & 0.87 & -44 & 98.7 & -11.36 \\
\hline $\begin{array}{l}\text { Sex B } \\
\text { DDO } 070\end{array}$ & 095723.1053421 & $\begin{array}{r}11.85 \\
0.14\end{array}$ & $\begin{array}{c}5.1 \times 3.5 \\
10\end{array}$ & 301 & 1 & 1.36 & .07 & 1.63 & 111 & 120.4 & -13.96 \\
\hline NGC 3109 & $100049.0-255504$ & $\begin{array}{r}10.39 \\
0.29\end{array}$ & $\begin{array}{c}19.7 \times 3.7 \\
10\end{array}$ & 403 & 1 & 1.33 & .08 & 1.71 & 110 & 143.9 & -15.52 \\
\hline $\begin{array}{l}\text { Antlia } \\
\text { PGC } 029194\end{array}$ & $100147.0-270521$ & $\begin{array}{r}16.19 \\
0.34\end{array}$ & $\begin{array}{c}2.0 \times 1.5 \\
10\end{array}$ & 361 & 9 & 1.32 & .06 & 1.70 & 65 & 144.7 & -9.75 \\
\hline $\begin{array}{l}\text { Sex A } \\
\text { DDO } 075\end{array}$ & $100829.5-042646$ & $\begin{array}{r}11.86 \\
0.19\end{array}$ & $\begin{array}{c}5.9 \times 4.9 \\
10\end{array}$ & 324 & 1 & 1.42 & .08 & 1.74 & 94 & 130.1 & -14.09 \\
\hline $\begin{array}{l}\text { UGC } 6817 \\
\text { DDO } 099\end{array}$ & 114816.8390930 & $\begin{array}{l}13.4 \\
0.11\end{array}$ & $\begin{array}{c}4.1 \times 1.5 \\
10\end{array}$ & 242 & 1 & 2.64 & .21 & 2.74 & 248 & 99.0 & -13.82 \\
\hline $\begin{array}{l}\text { KDG } 090 \\
\text { DDO } 113\end{array}$ & 121227.1362948 & $\begin{array}{r}15.40 \\
0.09\end{array}$ & $\begin{array}{c}1.5 \times 1.3 \\
-1:\end{array}$ & 284 & 6 & 2.86 & .14 & 2.98 & 286 & 102.7 & -11.87 \\
\hline NGC 4214 & 121308.2363620 & $\begin{array}{r}10.24 \\
0.09\end{array}$ & $\begin{array}{c}8.5 \times 6.6 \\
9\end{array}$ & 291 & 3 & 2.94 & .18 & 3.07 & 295 & 102.2 & -17.19 \\
\hline IC 3104 & $121545.0-792654$ & $\begin{array}{r}13.63 \\
1.70\end{array}$ & $\begin{array}{c}3.8 \times 1.8 \\
9\end{array}$ & 430 & 5 & 2.27 & .19 & 2.63 & 171 & 141.4 & -14.85 \\
\hline $\begin{array}{l}\text { UGC } 7577 \\
\text { DDO } 125\end{array}$ & 122515.4434613 & $\begin{array}{r}12.84 \\
0.09\end{array}$ & $\begin{array}{c}4.3 \times 2.4 \\
10\end{array}$ & 196 & 4 & 2.54 & .17 & 2.62 & 241 & 95.2 & -14.27 \\
\hline $\begin{array}{l}\text { GR } 8 \\
\text { DDO } 155\end{array}$ & 125610.9142914 & $\begin{array}{r}14.68 \\
0.11\end{array}$ & $\begin{array}{c}1.1 \times 1.0 \\
10\end{array}$ & 214 & 3 & 2.1 & .2 & 2.38 & 136 & 124.4 & -12.04 \\
\hline $\begin{array}{l}\text { UGC } 8508 \\
\text { I Zw060 }\end{array}$ & 132847.1551002 & $\begin{array}{r}14.40 \\
0.06\end{array}$ & $\begin{array}{c}1.7 \times 1.0 \\
10\end{array}$ & 62 & 5 & 2.56 & .15 & 2.55 & 186 & 83.3 & -12.70 \\
\hline UGC 8638 & 133658.5250144 & $\begin{array}{r}14.47 \\
0.06\end{array}$ & $\begin{array}{c}1.2 \times 0.8 \\
10\end{array}$ & 274 & 4 & 2.3 & .5 & 2.50 & 273 & 112.7 & -12.40 \\
\hline $\begin{array}{l}\text { UGC } 8651 \\
\text { DDO } 181\end{array}$ & 133744.2405931 & $\begin{array}{l}14.7 \\
0.03\end{array}$ & $\begin{array}{c}2.3 \times 1.3 \\
10\end{array}$ & 201 & 1 & 3.01 & .29 & 3.10 & 271 & 97.0 & -12.72 \\
\hline $\begin{array}{l}\text { E383-087 } \\
\text { PGC } 049050\end{array}$ & $134623.0-354848$ & $\begin{array}{r}11.03 \\
0.30\end{array}$ & $\begin{array}{c}4.5 \times 3.6 \\
8\end{array}$ & 326 & 1 & - & & - & 108 & 166.1 & \\
\hline KKH 86 & 135202.2042917 & $\begin{array}{l}16.8 \\
0.12\end{array}$ & $\begin{array}{c}0.7 \times 0.5 \\
10\end{array}$ & 283 & 3 & 2.61 & .16 & 2.92 & 205 & 131.6 & -10.40 \\
\hline KK 230 & 140501.5351809 & $\begin{array}{l}17.9 \\
0.06\end{array}$ & $\begin{array}{c}0.6 \times 0.5 \\
10\end{array}$ & 61 & 1 & 1.9 & .2 & 2.03 & 125 & 101.3 & -8.55 \\
\hline
\end{tabular}


Table 2. continued.

\begin{tabular}{|c|c|c|c|c|c|c|c|c|c|c|c|}
\hline Name & $\alpha(1950.0) \delta$ & $\begin{array}{l}B_{\mathrm{T}} \\
A_{B}\end{array}$ & $\begin{array}{c}a \times b \\
T\end{array}$ & $\overline{V_{\mathrm{h}}}$ & $\sigma$ & 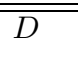 & $\bar{\sigma} \sigma$ & $\overline{D_{\mathrm{LG}}}$ & $\overline{V_{\mathrm{LG}}}$ & $\bar{\theta}$ & $M_{B}$ \\
\hline DDO 187 & 141338.6231713 & 14.38 & $1.7 \times 1.3$ & 154 & 4 & 2.5 & .2 & 2.70 & 174 & 112.2 & -12.71 \\
\hline UGC 9128 & & 0.10 & 10 & & & & & & & & \\
\hline UGC 9240 & 142248.4444504 & 13.25 & $1.8 \times 1.6$ & 150 & 4 & 2.79 & .26 & 2.84 & 266 & 91.3 & -14.03 \\
\hline DDO 190 & & 0.05 & 10 & & & & & & & & \\
\hline KKR 25 & 161237.3542946 & $\begin{array}{r}16.45 \\
0.04\end{array}$ & $\begin{array}{c}1.1 \times 0.9 \\
-1:\end{array}$ & -135 & 2 & 1.86 & .18 & 1.79 & $72:$ & 74.3 & -9.94 \\
\hline IC 4662 & $174212.0-643718$ & $\begin{array}{r}11.74 \\
0.30\end{array}$ & $\begin{array}{c}2.8 \times 1.6 \\
9\end{array}$ & 308 & 4 & - & & - & 145 & 132.3 & \\
\hline NGC 6789 & 191617.1635250 & $\begin{array}{r}13.76 \\
0.30\end{array}$ & $\begin{array}{c}1.3 \times 1.0 \\
10\end{array}$ & -141 & 9 & 3.6 & .20 & 3.33 & 144 & 50.1 & -14.32 \\
\hline SagDIG & $192705.4-174659$ & $\begin{array}{r}14.12 \\
0.49\end{array}$ & $\begin{array}{c}2.9 \times 2.1 \\
10\end{array}$ & -77 & 4 & 1.04 & .05 & 1.15 & 23 & 93.0 & -11.60 \\
\hline $\begin{array}{l}\text { DDO } 210 \\
\text { Aquarius }\end{array}$ & $204406.5-130155$ & $\begin{array}{l}14.0 \\
0.22\end{array}$ & $\begin{array}{c}2.2 \times 1.1 \\
10\end{array}$ & -137 & 5 & 0.94 & .04 & 0.94 & 13 & 76.7 & -11.09 \\
\hline IC 5152 & $215926.6-513214$ & $\begin{array}{r}11.06 \\
0.11\end{array}$ & $\begin{array}{c}5.2 \times 3.2 \\
10\end{array}$ & 124 & 3 & 2.07 & .18 & 2.19 & 75 & 98.9 & -15.63 \\
\hline $\begin{array}{l}\text { Tucana } \\
\text { PGC } 069519\end{array}$ & $223827.3-644054$ & $\begin{array}{l}15.7 \\
0.14\end{array}$ & $\begin{array}{c}2.9 \times 1.2 \\
-2\end{array}$ & 130: & 2 & 0.88 & .04 & 1.10 & 9: & 108.3 & -9.16 \\
\hline $\begin{array}{l}\text { E407-018 } \\
\text { UGCA } 438\end{array}$ & $232347.3-323950$ & $\begin{array}{r}13.86 \\
0.06\end{array}$ & $\begin{array}{c}1.5 \times 1.2 \\
10\end{array}$ & 62 & 5 & 2.23 & .15 & 2.17 & 99 & 75.7 & -12.94 \\
\hline KKH 98 & 234303.9382624 & $\begin{array}{l}16.7 \\
0.53\end{array}$ & $\begin{array}{c}1.1 \times 0.6 \\
10\end{array}$ & -136 & 3 & 2.45 & .13 & 2.02 & 152 & 11.2 & -10.78 \\
\hline $\begin{array}{l}\text { WLM } \\
\text { DDO } 221\end{array}$ & $235924.4-154422$ & $\begin{array}{r}11.03 \\
0.16 \\
\end{array}$ & $\begin{array}{c}11.5 \times 4.0 \\
9\end{array}$ & -116 & 2 & 0.92 & .04 & 0.78 & -10 & 57.5 & -13.95 \\
\hline SDIG & \multicolumn{11}{|c|}{ Laustsen et al. (1977). } \\
\hline NGC 55 & \multicolumn{11}{|c|}{ Puché \& Carignan (1988). } \\
\hline KK 3 & \multicolumn{11}{|c|}{ Karachentsev et al. (2000). } \\
\hline Cetus & \multicolumn{11}{|c|}{ Sarajedini et al. (2001). } \\
\hline NGC 247 & \multicolumn{11}{|c|}{ Puché \& Carignan (1988), Tammann (1987). } \\
\hline NGC 300 & \multicolumn{11}{|c|}{ Freedman et al. (1992), Soffner et al. (1996). } \\
\hline NGC 1569 & \multicolumn{11}{|c|}{ Karachentsev et al. (1994), Waller (1991). } \\
\hline UGCA 92 & \multicolumn{11}{|c|}{ Karachentsev et al. (1994). } \\
\hline Leo A & \multicolumn{11}{|c|}{ Tolstoy et al. (1998). } \\
\hline Antlia & \multicolumn{11}{|c|}{ Aparicio et al. (1997), $D=1.57 \pm 0.07 \mathrm{Mpc}$, Castellani et al. (2001). } \\
\hline Sex A & \multicolumn{11}{|c|}{ Sakai et al. (1996). } \\
\hline NGC 4214 & \multicolumn{11}{|c|}{ Maiz-Apellaniz et al. (2002), $D=2.67 \pm .20$, Drozdovsky et al. (2001a). } \\
\hline GR 8 & \multicolumn{11}{|c|}{ Dohm-Palmer et al. (1998). } \\
\hline UGC 8638 & \multicolumn{11}{|c|}{ Makarova et al. (1998). } \\
\hline KK 230 & \multicolumn{11}{|c|}{ Grebel \& Guhathakurta (2001). } \\
\hline DDO 187 & \multicolumn{11}{|c|}{ Aparicio et al. (2000). } \\
\hline KKR 25 & \multicolumn{11}{|c|}{$\begin{array}{l}\text { Karachentsev et al. ( } 2001 \mathrm{~b}) \text {; the heliocentric velocity of }-135 \mathrm{~km} \mathrm{~s}^{-1} \text {. } \\
\text { is probably caused by the Local HI. }\end{array}$} \\
\hline NGC 6789 & \multicolumn{11}{|c|}{ Drozdovsky et al. (2001b). } \\
\hline Tucana & \multicolumn{11}{|c|}{$\begin{array}{l}\text { Oosterloo et al. (1996); the heliocentric velocity of } 130 \mathrm{~km} \mathrm{~s}^{-1} \text {. } \\
\text { is probably caused by the Local HI. }\end{array}$} \\
\hline WLM & \multicolumn{11}{|c|}{ Dolphin (2000c). } \\
\hline
\end{tabular}

are of the same order. As a robust estimate of mass we used the relation

$M_{\text {orb }}=(32 / 3 \pi) \times G^{-1} \times\left(1-2 e^{2} / 3\right)^{-1}<R_{\mathrm{p}} \times \Delta V_{\mathrm{r}}^{2}>$, which is valid for arbitrarily-oriented Keplerian orbits with eccentricity $e$. Adopting $e=0.7$ as the average eccentricity, we derived the following mass estimates: $M(\mathrm{M} 81)=1.6 \times 10^{12} M_{\odot}$, and $M(\mathrm{CenA})=2.1 \times$ $10^{12} M_{\odot}$. For the galaxies M 31 and Milky Way the mass estimates from orbital motions of their companions are
$1.0 \times 10^{12} M_{\odot}(\mathrm{M} 31)$ and $1.0 \times 10^{12} M_{\odot}$ (Milky Way). In the case of the Milky Way it was taken into account that we use the actual spatial distances to the companions, $D$, instead of projected distances, $R_{\mathrm{p}}$. It should also be noted that a significant contribution to the Milky Way mass estimate is made by only one remote companion, Phoenix, which has a high peculiar velocity (Gallart et al. 2001). One can assume that the orbit of Phoenix does not fit the Keplerian motion because of its probable location in the "infall zone" (see Fig. 4). Then, removing Phoenix drops 


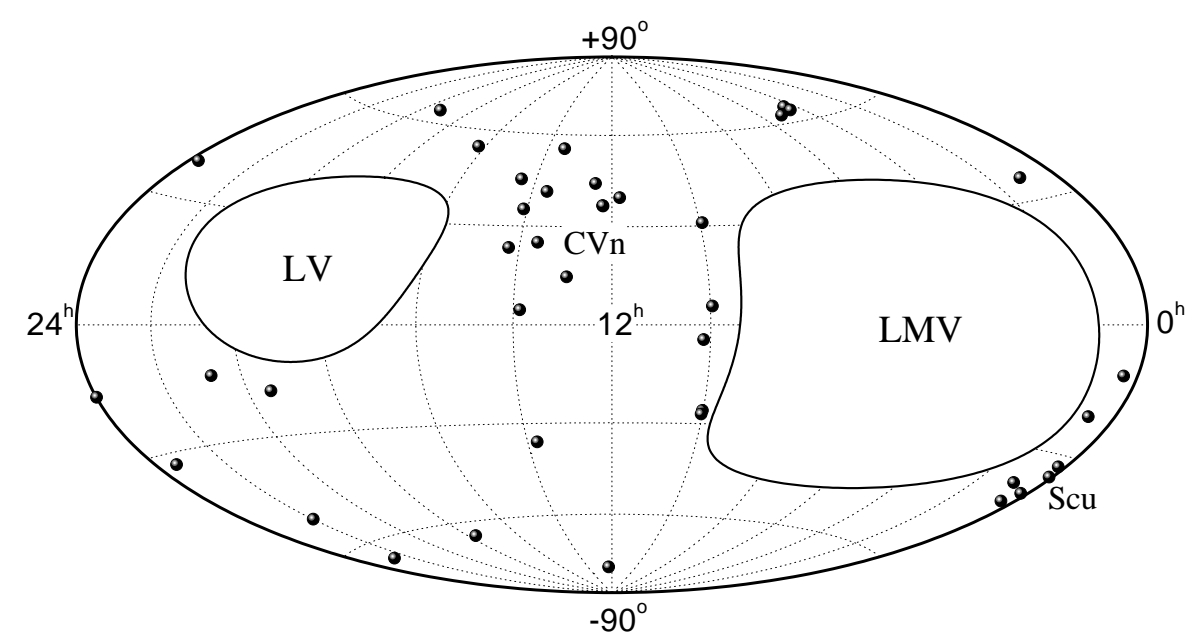

Fig. 3. All-sky distribution of the 38 galaxies around the LG in equatorial coordinates. The Local Void (LV) of Tully (1988) and the Local Minivoid (LMV) are outlined with solid lines.

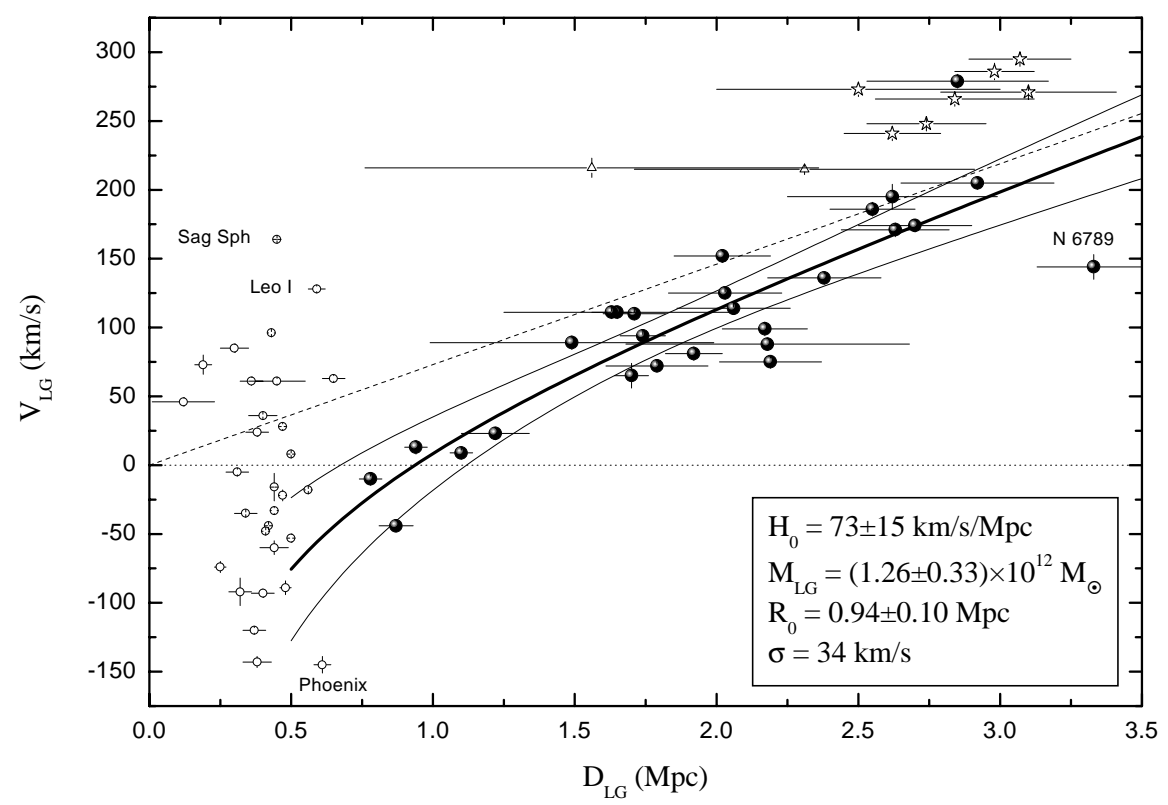

Fig. 4. Velocity-distance relation from the data in Table 2 (filled circles). Velocities and distances have been corrected to the centroid of the LG. The members of the LG from Table 3 are shown as open circles. Observational 1- $\sigma$ errors are indicated with bars. The thick solid line shows the velocity-distance regression with two parameters, $H_{0}=73 \mathrm{~km} \mathrm{~s} / \mathrm{Mpc}^{-1}$ and $M_{\mathrm{LG}}=$ $1.26 \times 10^{12} M_{\odot}$, derived as the least-square solution. The two thin solid lines correspond to the $95 \%$ confidence interval of the regression. The dashed straight line is an asymptotic Hubble relation with $H_{0}=73 \mathrm{~km} \mathrm{~s}^{-1} / \mathrm{Mpc}$. The seven probable members of the CVn cloud are shown as asterisks, and two galaxies, ScuDIG and NGC 247, with poorly determined distances are marked as triangles.

the Milky Way mass estimate to $0.5 \times 10^{12} M_{\odot}$, which seems to us more appropriate. The derived orbital masses are quite close to more sophisticated estimates of masses of the Milky Way and M 31 inside $\sim 50 \mathrm{Kpc:} M_{\mathrm{MW}}=$ $0.4 \times 10^{12} M_{\odot}($ Fich \& Tremaine 1991$), M_{\mathrm{MW}}=(0.5 \pm$ $0.1) \times 10^{12} M_{\odot}\left(\right.$ Kochanek 1996), $M_{\mathrm{M} 31}=0.8 \times 10^{12} M_{\odot}$ (Evans \& Wilkinson 2000), $M_{\mathrm{M}} 31=(0.8 \pm 0.1) \times 10^{12} M_{\odot}$ (Côté et al. 2000), and $M_{\mathrm{M}} 31=(0.7 \div 1.0) \times 10^{12} M_{\odot}$ (Evans et al. 2000). Therefore, the sum of orbital (virial) masses of both the LG subgroups, $(1.2 \div 1.5) \times 10^{12} M_{\odot}$, agrees within the errors with the total mass of the LG, $(1.3 \pm 0.3) \times 10^{12} M_{\odot}$, derived from the radius of the zero-velocity surface. The agreement of these mutually independent mass estimates corresponding to essentially different scale lengths, $\sim 50 \mathrm{kpc}$ and $\sim 1 \mathrm{Mpc}$, tell us that the major part of the mass in the LG is strongly concentrated towards its two biggest members.

\section{Concluding remarks}

The total view of the Local Group and its surroundings is presented in Fig. 5 in the Cartesian Supergalactic coordinates. The upper panel shows the Local Complex of galaxies seen in the Supergalactic plane. The nearby galaxies 
Table 3. Distances and radial velocities of the LG members.

\begin{tabular}{|c|c|c|c|c|c|}
\hline Name & Type & $\begin{array}{c}D \\
\mathrm{Mpc}\end{array}$ & $\begin{array}{l}V_{\mathrm{h}} \pm \sigma \\
\mathrm{km} \mathrm{s}^{-1}\end{array}$ & $\begin{array}{l}D_{\mathrm{LG}} \\
\mathrm{Mpc}\end{array}$ & $\begin{array}{r}V_{\mathrm{LG}} \\
\mathrm{km} \mathrm{s}^{-1}\end{array}$ \\
\hline IC 10 & 10 & 0.83 .05 & -3445 & 0.44 & -60 \\
\hline NGC 147 & -3 & 0.73 .05 & -1933 & 0.30 & 85 \\
\hline And III & -3 & 0.76 .04 & -35510 & 0.32 & -92 \\
\hline NGC 185 & -3 & 0.62 .03 & -2027 & 0.19 & 73 \\
\hline NGC 205 & -5 & 0.82 .04 & -2443 & 0.38 & 24 \\
\hline M 32 & -5 & 0.80 .04 & -2053 & 0.36 & 61 \\
\hline M 31 & 3 & 0.78 .04 & -3011 & 0.34 & -35 \\
\hline And I & -3 & 0.81 .04 & -3802 & 0.37 & -120 \\
\hline SMC & 10 & 0.06 .01 & 1584 & 0.47 & -22 \\
\hline Sculptor & -3 & 0.08 .01 & 1101 & 0.43 & 96 \\
\hline LGS 3 & $-1:$ & 0.62 .02 & -2864 & 0.25 & -74 \\
\hline IC 1613 & 10 & 0.73 .02 & -2325 & 0.48 & -89 \\
\hline And V & -3 & 0.81 .05 & -4034 & 0.38 & -143 \\
\hline And II & -3 & 0.53 .11 & -1883 & 0.12 & 46 \\
\hline M 33 & 5 & 0.81 .05 & -1801 & 0.40 & 36 \\
\hline Phoenix & $-1:$ & 0.44 .03 & -526 & 0.61 & -145 \\
\hline Fornax & -3 & 0.14 .01 & 532 & 0.44 & -33 \\
\hline LMC & 9 & 0.05 .005 & 2782 & 0.47 & 28 \\
\hline Carina & -3 & 0.10 .005 & 2233 & 0.50 & -53 \\
\hline Leo I & -3 & 0.25 .03 & 2852 & 0.59 & 128 \\
\hline Sex dSph & -3 & 0.086 .004 & 2261 & 0.50 & 8 \\
\hline Leo II & -3 & 0.21 .01 & 761 & 0.56 & -18 \\
\hline Ursa Min & -3 & 0.066 .003 & -2471 & 0.42 & -44 \\
\hline Draco & -3 & 0.086 .009 & -2931 & 0.41 & -48 \\
\hline Sgr dSph & -3 & 0.024 .002 & 1421 & 0.45 & 164 \\
\hline NGC 6822 & 10 & 0.49 .04 & -572 & 0.65 & 63 \\
\hline Cas dSph & -3 & 0.71 .04 & -3072 & 0.31 & -5 \\
\hline Pegasus & 10 & 0.76 .10 & -1822 & 0.45 & 61 \\
\hline Peg dSph & -3 & 0.78 .04 & -3543 & 0.40 & -93 \\
\hline Milky Way & 4 & 0.01 .002 & 010 & 0.44 & -16 \\
\hline LGS 3: & \multicolumn{5}{|c|}{ Miller et al. (2002). } \\
\hline Phoenix: & \multirow{2}{*}{\multicolumn{5}{|c|}{$\begin{array}{l}\text { Gallart C. et al. (2001). } \\
\text { mistake in } l, b \text {-coordinates } \\
\text { (Evans et al. 2000) is corrected. }\end{array}$}} \\
\hline Cas dSph: & & & & & \\
\hline IC 1613: & \multicolumn{5}{|c|}{ Dolphin et al. (2001). } \\
\hline
\end{tabular}

are represented as "illuminated balls", whose size is proportional to the galaxy luminosity. Spiral and irregular galaxies are indicated as "dark balls", and elliptical and spheroidal ones are shown as "light balls". The bottom panel presents the Local Complex from another point of view. The Canes Venatici cloud is situated on the right side, and the Sculptor group is located on the left side. The Local Minivoid occupies the bottom left corner, and the Local Void of Tully extends above the complex plane in the "+Z"-direction. Only one galaxy, NGC 6789 , is highly raised above the rest of the objects. The morphological segregation is evident: the dEs and gas-deficient dSphs are tightly concentrated around the large spirals.

Another manifestation of the morphological segregation can be seen from the data of Fig. 6, which presents the luminosity function (LF) of nearby galaxies. The upper histogram shows the absolute magnitude distribution for 38 "field" galaxies from Table 2. The bottom one corresponds to the combined LF of 96 galaxies in the LG, M 81 group and CenA group. E and dSph galaxies on the histograms are shaded. Because the effective depth of both the samples is almost the same, we have the first opportunity here to compare LFs of the field galaxies and the members of groups, avoiding strong bias effects. Comparison of the two histograms confirms the wellknown fact that in dense regions the LF has a steep shape (Binggeli et al. 1985; Ferguson \& Sandage 1990). However, this difference seems to be true for the integrated LF only, when all morphological types of galaxies are combined. Thus, the median absolute magnitude of $\mathrm{dSph}+\mathrm{dE}$ galaxies is approximately the same $\left(\sim-10^{\mathrm{m}} \cdot 7\right)$ for the field galaxies as well as for the members of the groups. The median absolute magnitudes of $\mathrm{dIrr}+\mathrm{S}$ galaxies are also similar $\left(\sim-14^{\mathrm{m}} 0\right)$ for both the samples. Because the relative number of $\mathrm{dSph}+\mathrm{dE}$ galaxies among the field objects is only $16 \%$, and their fraction among the group members is $53 \%$, the known effect of morphological segregation of galaxies leads to the observed difference in their LFs. Therefore, considering or ignoring gas-deficient dSphs may be the reason for a significant difference in LFs from one sample to another. For instance, the "blind" HIPASS survey (Banks et al. 1999) is insensitive to dSph galaxies, which renders the luminosity function of the HIPASS sample less steep than for optical surveys.

One can assume that the LF of field galaxies is the "initial" LF, being almost undistorted by interactions. In such a case the observed difference in LF for members of groups with respect to the field LF may be described as a result of two processes: a) formation of objects of high luminosity ("cannibals") due to merging of galaxies in groups, b) creation of new "tidal" dwarfs, say, via fragmentation of tidal bridges and tails (Duc et al. 2000).

As it has been emphasized by Governato et al. (1997) and Klypin et al. (2002), the dispersion of random motions of field galaxies and the centers of groups, $\sigma_{\mathrm{v}}$, contains key information about the scenario of galaxy formation and the value of the average density of matter, $\Omega_{\mathrm{m}}$. Sandage et al. (1972) noted that the velocity dispersion around the local Hubble flow is low $\left(\sigma_{v} \sim 70 \mathrm{~km} \mathrm{~s}^{-1}\right)$, which corresponds to $\Omega_{\mathrm{m}} \sim 0.1$. New observational data, based on much more accurate distance measurements, yield an essentially lower value of $\sigma_{v}$. Karachentsev et al. (2002a) showed that the peculiar velocity of the centroid of the M 81/NGC 2403 group does not exceed $35 \mathrm{~km} \mathrm{~s}^{-1}$. According to Karachentsev et al. (2002a), peculiar radial velocities of the centroids of the CenA and the M 83 groups are $+18 \pm 24 \mathrm{~km} \mathrm{~s}^{-1}$ and $-17 \pm 27 \mathrm{~km} \mathrm{~s}^{-1}$, respectively. From analysis of the local velocity field Karachentsev \& Makarov (2001) found the value and the direction of the solar apex with respect to the Local Volume galaxies with $D<7$ Mpc: $V_{\mathrm{a}}=(325 \pm 11) \mathrm{km} \mathrm{s}^{-1}, l_{\mathrm{a}}=(95 \pm 2)^{\circ}, b_{\mathrm{a}}=$ $(-4 \pm 1)^{\circ}$, where $l$ and $b$ are the galactic coordinates. Because the apex of solar motion with respect to the LG centroid is $V_{\mathrm{a}}=316 \mathrm{~km} \mathrm{~s}^{-1}, l_{\mathrm{a}}=93^{\circ}$, and $b_{\mathrm{a}}=-4^{\circ}$, the 


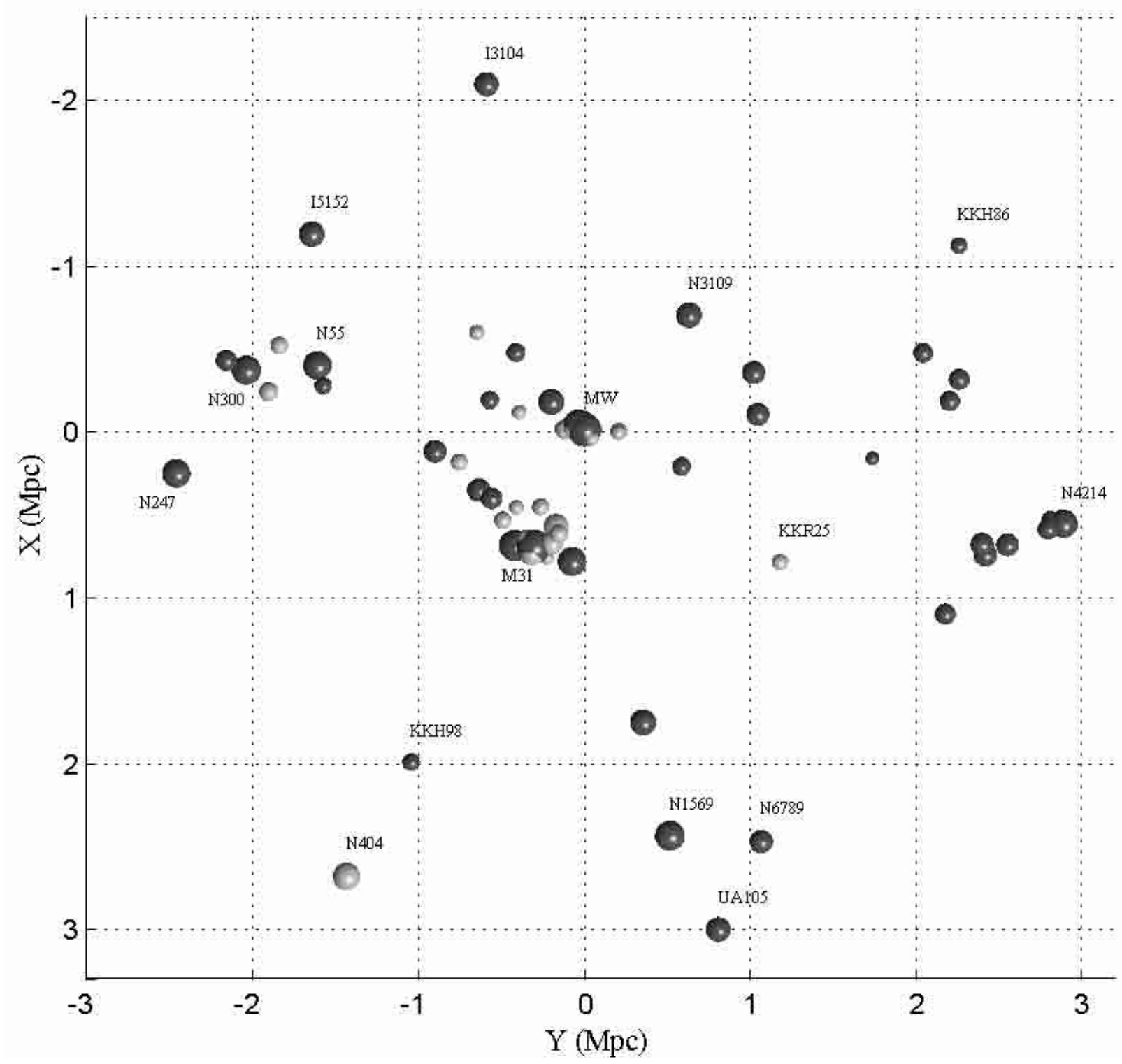

Fig. 5. The total view of the Local Group and its vicinity in the Cartesian Supergalactic coordinates. The upper panel shows the Local complex of galaxies seen in the Supergalactic plane. The nearby galaxies are represented as "iluminated balls", whose size is proportional to the galaxy luminosity. Spiral and irregular galaxies are indicated as dark balls, and elliptical and spheroidal ones are shown as light balls. The bottom panel presents the Local complex from another point of view. The Canes Venatici cloud is situated on the right side, and the Sculptor group is located on the left side. The Local Minivoid occupies the bottom left corner, and the Local Void of Tully extends above the complex plane in the "+Z"-direction.

peculiar velocity of the LG centroid with respect to the whole Local Volume turns out to be only about $10 \mathrm{~km} \mathrm{~s}^{-1}$.

Therefore, we conclude that the centroids of all nearby well-studied groups have surprisingly low dispersion of peculiar velocities, which is $\sigma_{v} \sim(20 \div 30) \mathrm{km} \mathrm{s}^{-1}$. The same value is also characteristic of the field galaxies in the vicinity of the LG. Baryshev et al. (2001) suggested that the observed quiescence of the local Hubble flow is a signature of the cosmological vacuum-dominated universe where the velocity perturbations are adiabatically decreasing.

Acknowledgements. We thank the referee,John Huchra, for his useful comments. Support for this work was provided by NASA through grant GO-08601.01-A from the Space Telescope
Science Institute, which is operated by the Association of Universities for Research in Astronomy, Inc., under NASA contract NAS5-26555. This work was partially supported by RFBR grant 01-02-16001 and DFG-RFBR grant 01-02-04006. D.G. acknowledges financial support for this project received from CONICYT through Fondecyt grant 8000002.

The Digitized Sky Surveys were produced at the Space Telescope Science Institute under U.S. Government grant NAG $\mathrm{W}-2166$. The images of these surveys are based on photographic data obtained using the Oschin Schmidt Telescope on the Palomar Mountain and the UK Schmidt Telescope. The plates were processed into the present compressed digital form with permission of these institutions.

This project made use of the NASA/IPAC Extragalactic Database (NED), which is operated by the Jet Propulsion 


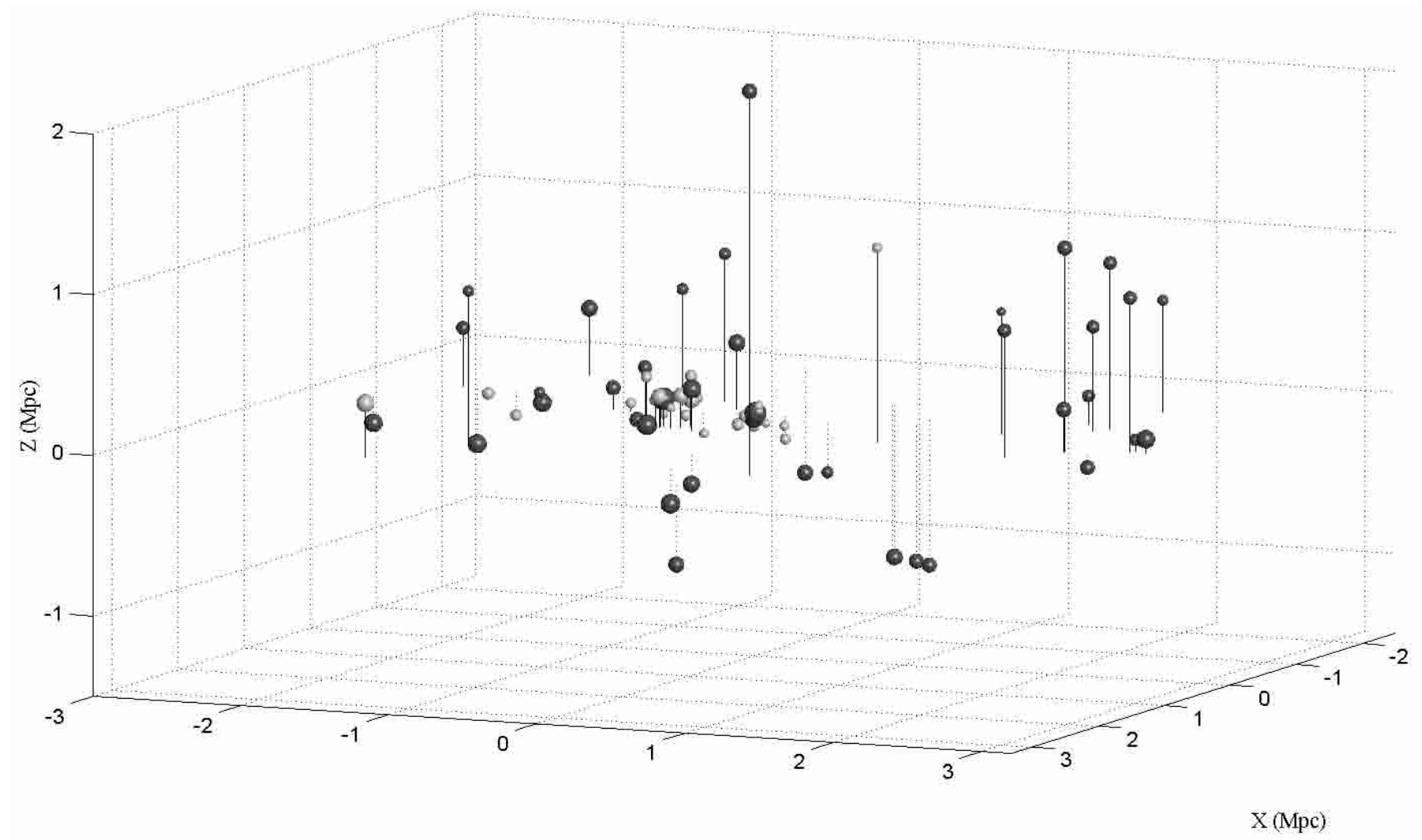

$\mathrm{Y}(\mathrm{Mpc})$

Fig. 5. continued.

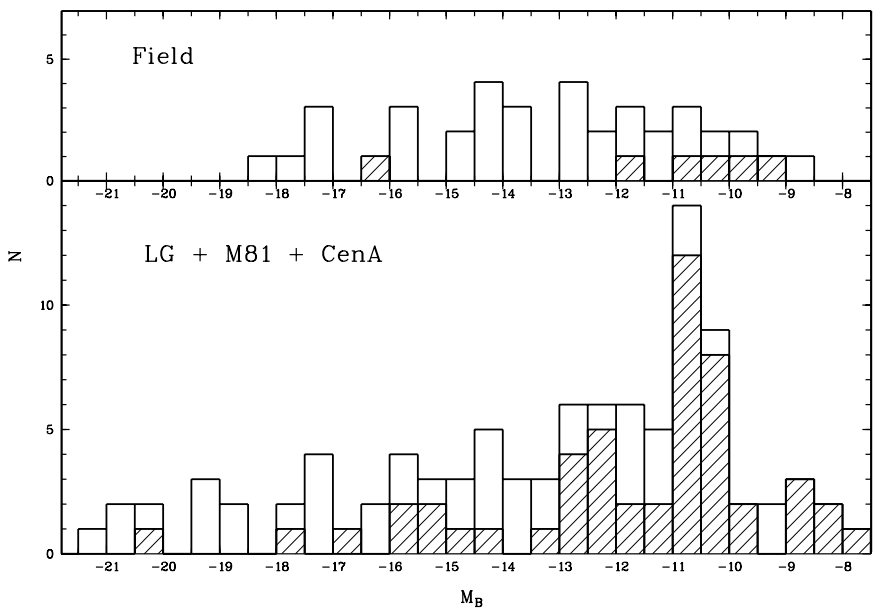

Fig. 6. The luminosity function of 38 nearby field galaxies from Table 2 (upper histogram) and 96 galaxies situated in the LG, the $\mathrm{M} 81$ group, and the CenA group. E and $\mathrm{dSph}$ galaxies are shaded.

Laboratory, Caltech, under contract with the National Aeronautics and Space Administration.

\section{References}

Aparicio, A., Dalcanton, J. J., Gallart, C., \& MartinezDelgado, D. 1997, AJ, 114, 1447

Aparicio, A., \& Tikhonov, N. 2000, AJ, 119, 2183
Aparicio, A., Tikhonov, N., \& Karachentsev, I. 2000, AJ, 119, 177

Banks, G. D., Disney, M. J., Knezek, P. M., et al. 1999, ApJ, 524,612

Baryshev, Yu., Chernin, A., \& Teerikorpi, P. 2001, A\&A, 378, 729

Bellazzini, M., Ferraro, F. R., \& Pancino, E. 2001, ApJ, 556, 635

Binggeli, B., Sandage, A., \& Tammann, G. A. 1985, AJ, 90, 1681

Capaccioli, M., Piotto, G., \& Bresolin, F. 1992, AJ, 103, 1157

Castellani, M., Bono, G., Cassisi, S., Marconi, G., \& Piersimoni, A. 2001 [astro-ph/0008080]

Côté Mateo, M., Sargent, W. L. W., \& Olszewski, E. W. 2000, ApJ, 537, L91

Da Costa, G. S., \& Armandroff, T. E. 1990, AJ, 100, 162

Dohm-Palmer, R. C., Skillman, E .D., Gallagher, J., et al. 1998, AJ, 116, 1227

Dolphin, A. E. 2000a, PASP, 112, 1383

Dolphin, A. E. 2000b, PASP, 112, 1397

Dolphin, A. E. 2000c, ApJ, 531, 804

Dolphin, A. E., Saha, A., Skillman, E. D., et al. 2001, ApJ, 550,554

Drozdovsky, I. O., Schulte-Ladbeck, R. E., Hopp, U., Greggio, L., \& Crone, M. 2001a, in Dwarf Galaxies and their Environment, ed. K. S. Boer, R. Dettmar, \& U. Klein (Bad Honnef, Shaker Verlag, Aachen), 107

Drozdovsky, I. O., Schulte-Ladbeck, R. E., Hopp, U., Greggio, L., \& Crone, M. 2001b, in Dwarf Galaxies and their Environment, ed. K. S. Boer, R. Dettmar, \& U. Klein (Bad Honnef, Shaker Verlag, Aachen), 95 
Duc, P. A., Brinks, E., Springer, V., Pichardo, B., Wellebacher, P., \& Mirabel, I. F. 2000, AJ, 120, 1238

Evans, N. W., Wilkinson, M. I., Guhathakurta, P., Grebel, E. K., \& Vogt, S. S. 2000, ApJ, 540, L9

Evans, N. W., \& Wilkinson, M. I. 2000, MNRAS, 316, 929

Ferguson, H. C., \& Sandage, A. 1990, AJ, 100, 1

Ferrarese, L., et al. 2000, ApJ, 529, 745

Fich, M., \& Tremaine, S. 1991, ARA\&A, 29, 409

Freedman, W. L., Madore, B. F., Hawley, S. L., et al. 1992, ApJ, 396, 80

Gallart, C., Martinez-Delgado, D., Gomez-Flechoso, M. A., \& Mateo, M. 2001, AJ, 121, 2572

Georgiev, T. B., Karachentsev, I. D., \& Tikhonov, N. A. 1997, Lett. Astron. Zh., 23, 586

Giraud, E. 1986, A\&A, 170, 1

Giraud, E. 1990, A\&A, 231, 1

Governato, F., Moore, B., Cen, R., et al. 1997, New Astron., 2,91

Grebel, E. K., \& Guhathakurta, P. 2001, private communication

Jerjen, H., Freeman, K. C., \& Binggeli, B. 1998, AJ, 116, 2873

Jerjen, H., Rekola, R., Takalo, L., Coleman, M., \& Valtonen, M. 2001, A\&A, 380, 90

Karachentseva, V. E., \& Karachentsev, I. D. 1998, A\&AS, 127, 409

Karachentseva, V. E., Karachentsev, I. D., \& Richter, G. M. 1999, A\&AS, 135, 221

Karachentsev, I. D., Tikhonov, N. A., \& Sazonova, L. N. 1994, Lett. Astron. Zh., 20, 104

Karachentsev, I. D., Kopylov, A. I., \& Kopylova, F. G. 1994, Bull. Spec. Astrophys. Obs., 38, 5

Karachentsev, I., \& Makarov, D. 1996, AJ, 111, 535

Karachentsev, I., \& Musella, I. 1996, A\&A, 315, 348

Karachentsev, I., Drozdovsky, I., Kajsin, S., et al. 1997, A\&AS, 124,559

Karachentsev, I., \& Makarov, D. 1999, in Galaxy interactions at low and high redshifts, ed. J. E. Barnes, \& D. B. Sanders, Netherlands, 109

Karachentsev, I. D., Aparicio, A., \& Makarova, L. N. 1999, A\&A, 352, 363

Karachentsev, I. D., Sharina, M .E., Grebel, E. K., et al. 2000, ApJ, 542, 128

Karachentsev, I. D., \& Makarov, D. I. 2001, Astrofizika, 44, 5

Karachentsev, I. D., Karachentseva, V. E., \& Huchtmeier, W. K. 2001a, A\&A, 366, 428

Karachentsev, I. D., Sharina, M. E., Dolphin, A. E., et al. 2001b, A\&A, 379, 407

Karachentsev, I. D., Dolphin, A. E., Geisler, D., et al. 2002a, A\&A, 383, 125

Karachentsev, I. D., Sharina, M. E., Dolphin, A. E., et al. 2002b, A\&A, 385, 21

Klypin, A. A., Hoffman, Y., Kravtsov, A. V., \& Gottlober, S. 2002 , in press [astro-ph/0107104]

Kochanek, C. S. 1996, ApJ, 457, 228

Laustsen, S., Richter, W., van der Lans, J., et al. 1977, A\&A, 54,639

Lee, M. G., Freedman, W. L., \& Madore, B. F. 1993, ApJ, 417, 553

Lee, M. G., Aparicio, A., Tikhonov, N., et al. 1999, AJ, 118, 853
Lee, M. G., \& Byun, Y. 1999, AJ, 118, 817

Lee, M. G., \& Kim, S. C. 2000, AJ, 119, 777

Lynden-Bell, D. 1981, Observatory, 101, 111

Maiz-Apellaniz, J., Cieza, L., \& MacKenty, J. W. 2002, AJ, 123,1307

Makarova, L. N., Karachentsev, I. D., \& Georgiev, T. B. 1997, Astron. Lett., 23, 378

Makarova, L. N., Karachentsev, I. D., Takalo, L., et al. 1998, A\&AS, 128, 459

Makarova, L. N., Karachentsev, I. D., Grebel, E. K., \& Barsunova, O. Yu. 2002, A\&A, 384, 72

Mendez, B., Davis, M., Moustakas, J., et al. 2002 [astro-ph/0204192]

Miller, B. W., Dolphin, A. E., Lee, M. G., Kim, S. C., \& Hodge, P. 2002, ApJ, 562, 713

Oosterloo, T., Da Costa, G. S., \& Staveley-Smith, L. 1996, AJ, 112, 1969

Puche, D., \& Carignan, C. 1988, AJ, 95, 1025

Sakai, S., Madore, B. F., \& Freedman, W. L. 1996, ApJ, 461, 713

Sakai, S., Madore, B. F., \& Freedman, W. L. 1997, ApJ, 480, 589

Salaris, M., \& Cassisi, S. 1997, MNRAS, 289, 406

Sandage, A., Tammann, G. A., \& Hardy, E. 1972, ApJ, 172, 253

Sandage, A. 1986, ApJ, 307, 1

Sandage, A. 1987, ApJ, 317, 557

Sarajedini, A., Grebel, E. K., Dolphin, A. E., et al. 2002, ApJ, 567,915

Schlegel, D. J., Finkbeiner, D. P., \& Davis, M. 1998, ApJ, 500, 525

Seitzer, P., Grebel, E. K., Dolphin, A. E., et al. 1999, BAAS, $195,8.01$

Soffner, T., Mendez, R. H., Jacoby, G. H., et al. 1996, A\&A, 306,9

Stephens, A. W., Frogel, J. A., Freedman, W., et al. 2001, AJ, 121,2584

Tammann, G. A. 1987, in IAU Symp. 124, ed. A. Hewitt, G. Burbidge, \& L. Fang (Dordrecht, Kluwer), 151

Tikhonov, N. A., Karachentsev, I. D., Bilkina, B. I., \& Sharina, M. E. 1992, A\&A Trans., 1, 269

Tikhonov, N. A., \& Karachentsev, I. D. 1998, A\&AS, 128, 325

Tolstoy, E., Gallagher, J. S., Cole, A. A., et al. 1998, AJ, 116, 1244

Tonry, J. L., Dressler, A., Blakeslee, J. P., et al. 2001, ApJ, 546,681

Tully, R. B., \& Fisher, J. R. 1977, A\&A, 54, 661

Tully, R. B. 1988, Nearby Galaxy Catalog (Cambridge Univ. Press)

Tully, R. B., Somerville, R. S., Trentham, N., \& Verheijen, M. A. 2002, ApJ, 569, 573

Udalski, A., Wyrzykowski, L., Pietrzynski, G., et al. 2001, Acta Astron., 51, 221

van den Bergh, S. 2000, The galaxies of the Local Group (Cambridge Univ. Press)

Waller, H. 1991, ApJ, 370, 144

Zijlstra, A. A., \& Minniti, D. 1999, AJ, 117, 1743 